Research Article

\title{
Guided Wave Propagation for Monitoring the Rail Base
}

\author{
Guodong Yue $\mathbb{D}^{1},{ }^{1}$ Xiushi Cui, ${ }^{1}$ Ke Zhang, ${ }^{2}$ Zhan Wang, ${ }^{1}$ and Dong An $\mathbb{D}^{1}$ \\ ${ }^{1}$ School of Mechanical Engineering, Shenyang Jianzhu University, Shenyang, Liaoning 110168, China \\ ${ }^{2}$ Joint International Research Laboratory of Modern Construction Engineering Equipment and Technology, Shenyang, \\ Liaoning 110168, China \\ Correspondence should be addressed to Guodong Yue; ygd@sjzu.edu.cn and Dong An; andong@sjzu.edu.cn
}

Received 13 June 2020; Accepted 14 July 2020; Published 8 August 2020

Academic Editor: Gaetano Giunta

Copyright ( 2020 Guodong Yue et al. This is an open access article distributed under the Creative Commons Attribution License, which permits unrestricted use, distribution, and reproduction in any medium, provided the original work is properly cited.

\begin{abstract}
In order to monitor the rail base, the dispersion characteristics and propagation properties of the guided wave are studied. Firstly, two modes named as Modes $V_{1}$ and $V_{2}$ are selected by the semianalytical finite element method (SAFE). The region at the bottom edge can be monitored by Mode $V_{1}$, while the junction of the base edge and the flange can be detected by Mode $V_{2}$. Then, the characteristics in the propagation process are analyzed using the finite element method (FEM). The two modes can be separated about $0.6 \mathrm{~ms}$ after they are excited. Thirdly, a wave attenuation algorithm based on mean is proposed to quantify the wave attenuation. Both waves can have weak attenuation and be detected within $5 \mathrm{~m}$. Finally, a mode-identified experiment is performed to validate the aforementioned analysis. And a defect detection experiment is performed to demonstrate the excellent monitoring characteristics using Mode $V_{2}$. These results can be used to monitor the rail base in practice engineering.
\end{abstract}

\section{Introduction}

High speed railways are crucial infrastructures and are developing rapidly in a host of countries. With the increase in train speed, wheel-rail dynamic impact forces become increasingly intense. This makes rail suffer from alternating load with large peaks and short periods [1]. Besides, rails lie in the wild, encounter a large temperature difference day and night, and endure high-temperature stress $[2,3]$. These make rail prone to failure and cause serious accidents. Because the rail base is at the lower part of the rail and is affected by the sleepers, it has been and still a crucial research issue to monitor the safe status of rail base [4].

The rail is a highly directional structure and is extremely suitable for guided wave inspection. The guided wave-based method believes that rail damage can induce changes in propagation characteristics of the guided wave (reflected wave, transmitted wave, energy, etc.) [5-7]. The excitation sensors are arranged on one side of the rail with the receiving sensors on the same side or the other side. By studying the transmission time and energy, it can be judged whether the damage exists, the location and severity of the damage in the rail $[8,9]$. Because the guided wave can travel a long distance in the rail, this method can monitor the status of a section of the rail over a long distance. However, due to the irregular cross section of the rail, a sea of new guided wave modes with different dispersion characteristics appears during their propagation. In engineering applications, it is only indispensable to monitor changes in one or some modes in a certain frequency interval. This requires us to study the characteristics of the guided wave and select the reasonable mode.

Researchers use the semianalytical finite element method (SAFE) $[10,11]$ and the finite element method (FEM) to distinguish propagation characteristics (wave structure, dispersion curve, etc.) of these waves [12-16]. In the FEM, time domain simulation is performed on a three-dimensional model. The element size should be less than the onetenth of the wavelength to be studied. It makes element numbers extremely huge. This is computationally expensive. The mode shapes and wavelength are inspected manually to determine which mode of propagation they correspond to. Then, the phase velocity can be obtained. The phase velocity curve can be plotted. If two phase velocity curves intersect, phase velocity curves are arduous to be estimated by a finite difference scheme. The SAFE obtains wave propagation 
characteristics by studying the variation of the structural cross section. This method transforms a three-dimensional problem into a two-dimensional problem. The length of the waveguide is treated analytically. Most of the computations concentrate on the solution of the eigenvalues and corresponding eigenvectors to picture different modes [17]. These greatly improve the calculation efficiency. The results can be used directly in the two dimensions, without significant postprocessing by the user. But the process of the wave propagation cannot be demonstrated. The two methods are combined to get the whole information about the guided wave. Hence, the dispersion characteristics are obtained by the SAFE. And the propagation properties are demonstrated by the FEM in this paper.

In the wave propagation process, energy attenuation will be caused by structural damping, interface reflection, etc. Obviously, those guided waves that decay slowly should be selected as the target mode. When a wave velocity is constant, studying the wave attenuation can be converted into a comparison of the particle displacement at the corresponding positions in the two planes along the wave propagation direction. If the displacement difference is less, the energy attenuation is less; otherwise, the energy attenuation is larger. Statistical analysis of the comparison results can become an index to quantify the degree of wave attenuation. According to this idea, a wave attenuation algorithm based on mean is proposed to quantify the wave attenuation.

In order to use ultrasonic guided waves to monitor the rail base, the paper is organized as follows. To begin with, Section 2 will provide a brief background on the calculation process of the SAFE and elaborate the proposed wave attenuation algorithm based on mean. Section 3 demonstrates the use of the SAFE and the FEM in the analysis of the dispersion characteristics and the propagation properties and calculates the wave attenuation for two typical modes. A mode-identified experiment and a defect detection experiment are carried out in Section 4. Finally, a conclusion is given in Section 5.

\section{Theoretical Basis}

2.1. Semianalytical Finite Element Method. The semianalytical finite element method is widely used to solve the propagation characteristics of guided waves in arbitrary but constant cross-sectional waveguide's medium. It discretizes the cross section into a sea of finite element meshes. A complex exponential function is used in the two-dimensional element to represent the wave motion along the waveguide. An analytical method is used to solve the guided waves in the propagation direction. Triangular elements are used to conduct the geometric dispersion of the waveguides of cross sections. And the displacement of any node in the model can be expressed as

$$
u=\left[\begin{array}{lll}
u_{x} & u_{y} & u_{z}
\end{array}\right]^{T},
$$

where $u_{x}, u_{y}$, and $u_{z}$ represent the displacements in the $x$-, $y$-, and $z$-direction, respectively. And the symbol $[*]^{T}$ represents the transpose.
The displacement propagated is of the following form:

$$
\begin{aligned}
& u_{x}(x, y, z, t)=u_{x}(x, y) e^{-j(k z-\omega t)}, \\
& u_{y}(x, y, z, t)=u_{y}(x, y) e^{-j(k z-\omega t)}, \\
& u_{z}(x, y, z, t)=u_{z}(x, y) e^{-j(k z-\omega t)} .
\end{aligned}
$$

The stress and strain can be expressed as

$$
\begin{aligned}
\sigma & =\left[\begin{array}{llllll}
u_{x x} & u_{y y} & u_{z z} & u_{y z} & u_{z x} & u_{x y}
\end{array}\right]^{T}, \\
\varepsilon & =\left[\begin{array}{llllll}
\varepsilon_{x x} & \varepsilon_{y y} & \varepsilon_{z z} & \varepsilon_{y z} & \varepsilon_{z x} & \varepsilon_{x y}
\end{array}\right]^{T},
\end{aligned}
$$

where $u_{i j}$ and $\varepsilon_{i j}$ represent the stress and strain in the $i$ and $j$ plane, respectively. And when $i$ is not equal to $j$, they represent the shear stress and the shear strain, respectively.

The relationship between stress and strain vector is

$$
\sigma=C \varepsilon,
$$

where $C$ is the material stiffness matrix of the element when the research object is an isotropic material.

The relationship between displacement and strain vector is

$$
\varepsilon=\left[L_{x} \frac{\partial}{\partial x}+L_{y} \frac{\partial}{\partial y}+L_{z} \frac{\partial}{\partial z}\right] u,
$$

where

$$
\begin{aligned}
L_{x} & =\left[\begin{array}{lll}
1 & 0 & 0 \\
0 & 0 & 0 \\
0 & 0 & 0 \\
0 & 0 & 0 \\
0 & 0 & 1 \\
0 & 1 & 0
\end{array}\right], \\
L_{y} & =\left[\begin{array}{lll}
0 & 0 & 0 \\
0 & 1 & 0 \\
0 & 0 & 0 \\
0 & 0 & 1 \\
0 & 0 & 0 \\
1 & 0 & 0
\end{array}\right], \\
L_{z} & =\left[\begin{array}{lll}
0 & 0 & 0 \\
0 & 0 & 0 \\
0 & 0 & 1 \\
0 & 1 & 0 \\
1 & 0 & 0 \\
0 & 0 & 0
\end{array}\right],
\end{aligned}
$$

According to Hamilton's principle, the wave governing equation of each element can be obtained as

$$
\left(K_{1}+i k K_{2}+k^{2} K_{3}-\omega^{2} M\right) Q=0,
$$

where $K_{1}, K_{2}$, and $K_{3}$ are the stiffness matrices of the element, $M$ is the mass matrix, and $Q$ is the displacement vector of the element node. The imaginary term is included in the formula. To simplify the equation solution, the unitary transformation matrix $T$ is introduced: 


$$
T=\left[\begin{array}{lllllll}
i & & & & & & \\
& 1 & & & & & \\
& & 1 & & & & \\
& & & \ddots & & & \\
& & & i & & \\
& & & & 1 & \\
& & & & & 1
\end{array}\right] .
$$

And the following transformations are done:

$$
\begin{aligned}
\widehat{K}_{2} & =i T^{T} K_{2} T, \\
\widehat{Q} & =T Q .
\end{aligned}
$$

Equation (8) can be transformed into

$$
\left(K_{1}+k \widehat{K}_{2}+k^{2} K_{3}-\omega^{2} M\right) \widehat{Q}=0 .
$$

For each wavenumber $k$, the value of the characteristic frequency can be solved. Thereby, the frequency-wavenumber relationship of the waveguide can be obtained.

For solving the wave structure, (12) can be reexpressed as a first-order eigensystem:

$$
[A-k B]\left[\begin{array}{c}
\widehat{Q} \\
k \widehat{Q}
\end{array}\right]=0,
$$

where $\quad A=\left[\begin{array}{cc}0 & K_{1}-\omega^{2} M \\ K_{1}-\omega^{2} M & \widehat{K}_{2}\end{array}\right]$ and $B=\left[\begin{array}{cc}K_{1}-\omega^{2} M & 0 \\ 0 & -K_{3}\end{array}\right]$.

By solving (13), the displacement field vectors corresponding to the eigenvalues of each model can be obtained, that is, wave structures of the waveguides.

2.2. Wave Attenuation Algorithm Based on Mean. Assuming a node displacement in a cross section along the wave propagation direction is $u_{i}$, the mean value of node displacements in the mode wave can be expressed as

$$
\bar{u}=\frac{1}{N} \sum_{i=1}^{N} u_{i}
$$

where $N$ is the total number of nodes in the cross section in the mode wave.

The mode wave can be identified by the following sequence ID:

$$
\operatorname{ID}(i)= \begin{cases}1, & u_{i} \geq \bar{u} \\ 0, & u_{i}<\bar{u}\end{cases}
$$

ID can be also expressed in hexadecimal for simplicity.

The attenuation of the mode wave along the propagation direction can be calculated by the following steps:

(1) Calculate the mode wave identifications in two cross sections along the wave propagation direction. The two cross sections are indicated by the letters "a" and "b." Their node displacements are $u_{i}^{a}$ and $u_{i}^{b}$, respectively. Their identifications can be obtained by

$$
\begin{gathered}
\operatorname{ID}_{a}(i)= \begin{cases}1, & u_{i} \geq \bar{u}, \\
0, & u_{i}<\bar{u},\end{cases} \\
\operatorname{ID}_{b}(i)= \begin{cases}1, & u_{i} \geq \bar{u}, \\
0, & u_{i}<\bar{u},\end{cases} \\
\bar{u}=\frac{1}{N} \sum_{i=1}^{N} u_{i}^{a} .
\end{gathered}
$$

(2) Calculate the difference between two sequences with XOR operation:

$$
\operatorname{Dif}\left(\mathrm{ID}_{a}, \mathrm{ID}_{b}\right)=\mathrm{ID}_{a} \oplus \mathrm{ID}_{b} .
$$

(3) Calculate the hamming distance of the two sequences. Count the number as $D\left(\mathrm{ID}_{a}, \mathrm{ID}_{b}\right)$ of occurrences of 1 in the $\operatorname{Dif}\left(\mathrm{ID}_{a}, \mathrm{ID}_{b}\right)$ :

$$
D\left(\mathrm{ID}_{a}, \mathrm{ID}_{b}\right)=\operatorname{sum}\left(\text { occurrence of } 1 \text { in } \operatorname{Dif}\left(\mathrm{ID}_{a}, \mathrm{ID}_{b}\right)\right) \text {. }
$$

The hamming distance $D\left(\mathrm{ID}_{a}, \mathrm{ID}_{b}\right)$ represents the degree of attenuation. The larger the value, the greater the attenuation. If the hamming distance is no larger than 10 , the wave has weak attenuation.

\section{Vertical Mode of Guided Wave in Rail Base}

3.1. Propagation Characteristics of Vertical Mode of Guided Wave. The CHN60 rail is used for high-speed railway in China. According to the GB-2585-2007 standard, its model was established with height of $0.176 \mathrm{~m}$, width of $0.15 \mathrm{~m}$, and rail base edge thickness of $0.012 \mathrm{~m}$, as demonstrated in Figure 1. The propagation characteristics of guided waves in rail were calculated by SAFE method. Its material properties were set as follows: density $\left(\rho=7800 \mathrm{~kg} / \mathrm{m}^{3}\right)$, Young's modulus $(E=205.9 \mathrm{GPa})$, and Poisson's ratio $(\mu=0.3)$. Triangular elements were used to discretize the geometric cross sections of the rail, and the maximum mesh size was $0.007 \mathrm{~m}$.

Figure 2 demonstrates the wave-frequency dispersion curves of guided waves of $\mathrm{CHN} 60$ rails in a frequency band of $0-100 \mathrm{kHz}$ by SAFE method. The number of guided wave modes in rails increases sharply with the increase of frequency. These modes can be divided into three guided waves, including transverse modes, vertical modes, and longitudinal modes based on the excitation direction [18]. Guided wave transducers are usually deployed on the upper surface of rail base flange during the nondestructive testing of rail base. Because the geometry of the track base can be used to easily excite the vertical modes, the vertical modes are selected for research.

Figure 3 demonstrates the dispersion curves of two vertical guided wave modes for the phase and group velocities named Mode $V_{1}$ and Mode $V_{2}$, respectively. Most of their energy is concentrated at the rail base. When the frequency is lower than $40 \mathrm{kHz}$, their group and phase velocities are more sensitive to frequency change. When the frequency is higher than $80 \mathrm{kHz}$, the group and phase velocities tend to be stable. 


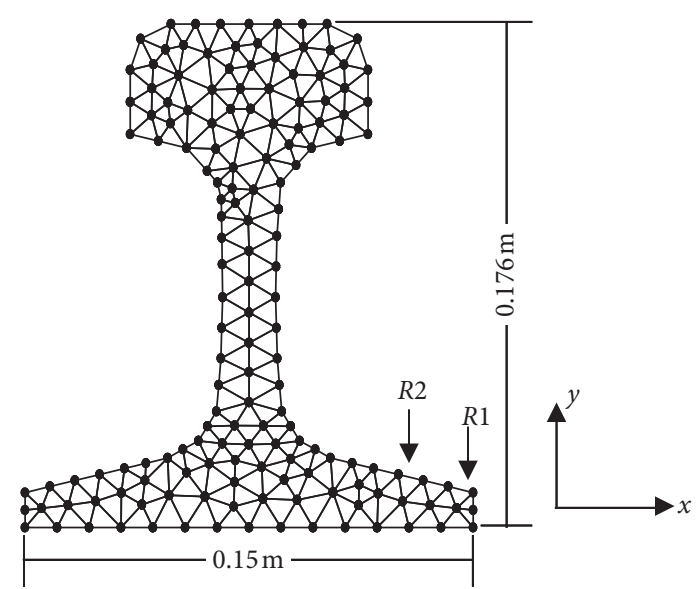

FIgURE 1: CHN60 rail geometry and meshing.

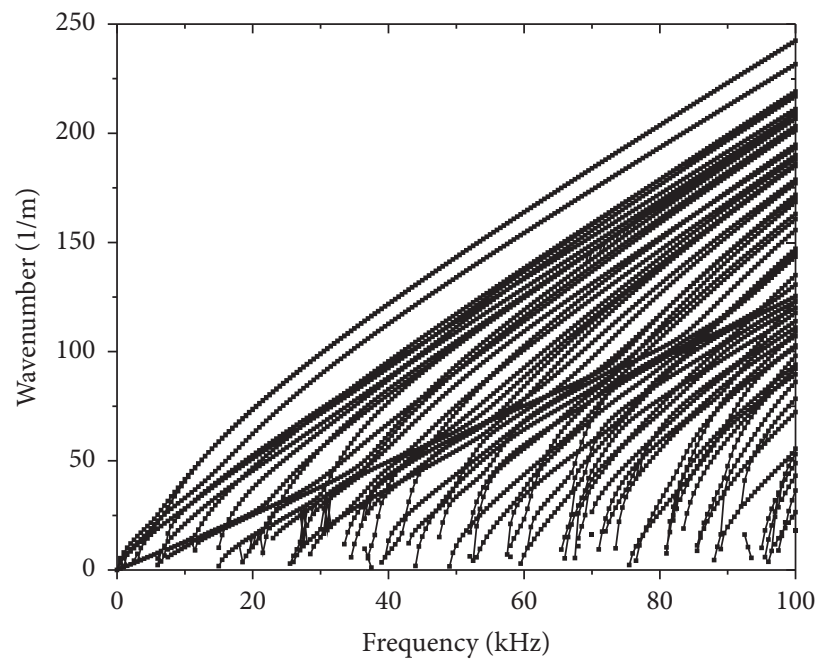

Figure 2: Dispersion curve of CHN60 rail by SAFE method.

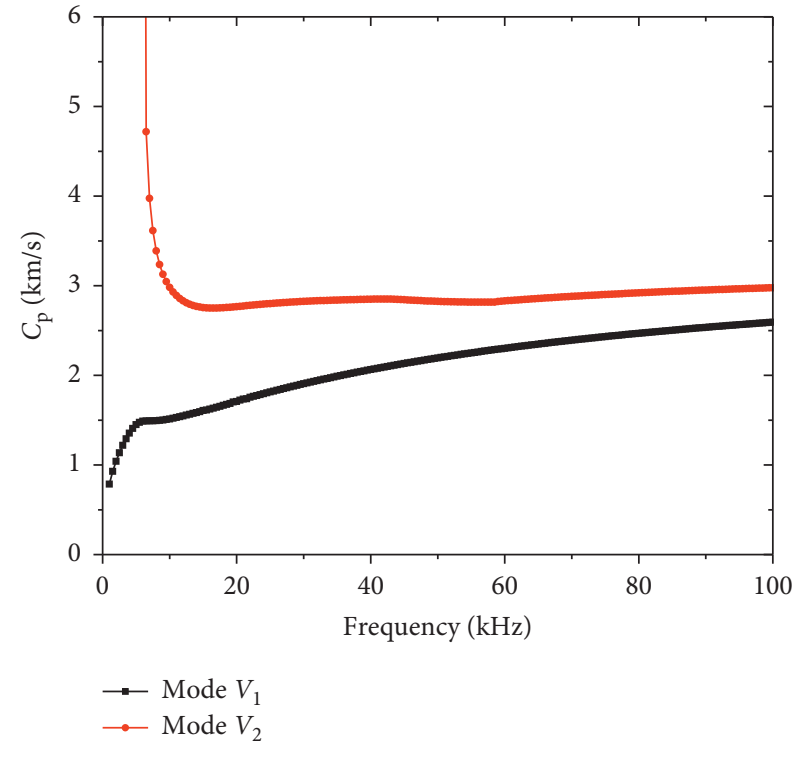

(a)

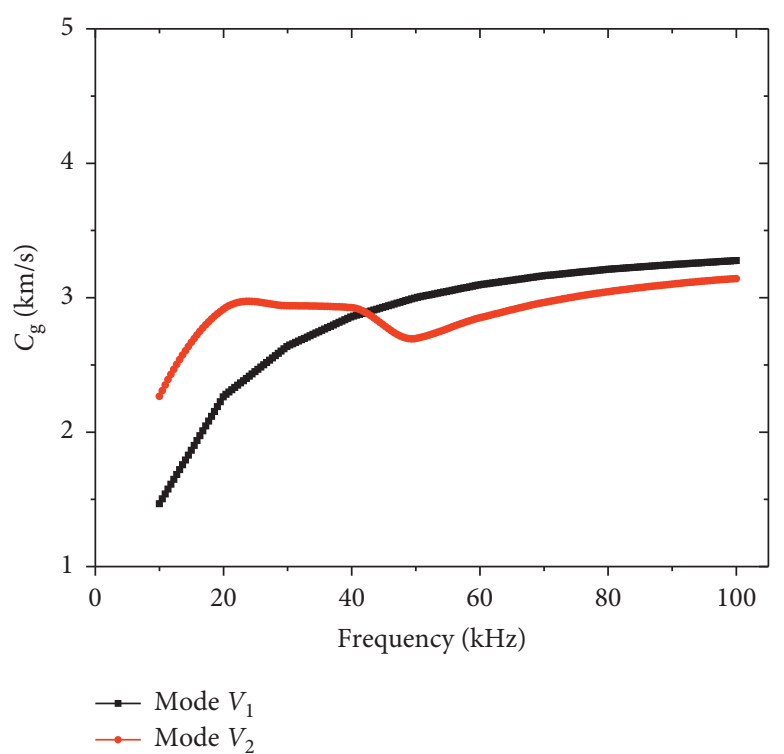

(b)

Figure 3: The vertical modes for CHN60 rail. (a) Phase velocity. (b) Group velocity. 


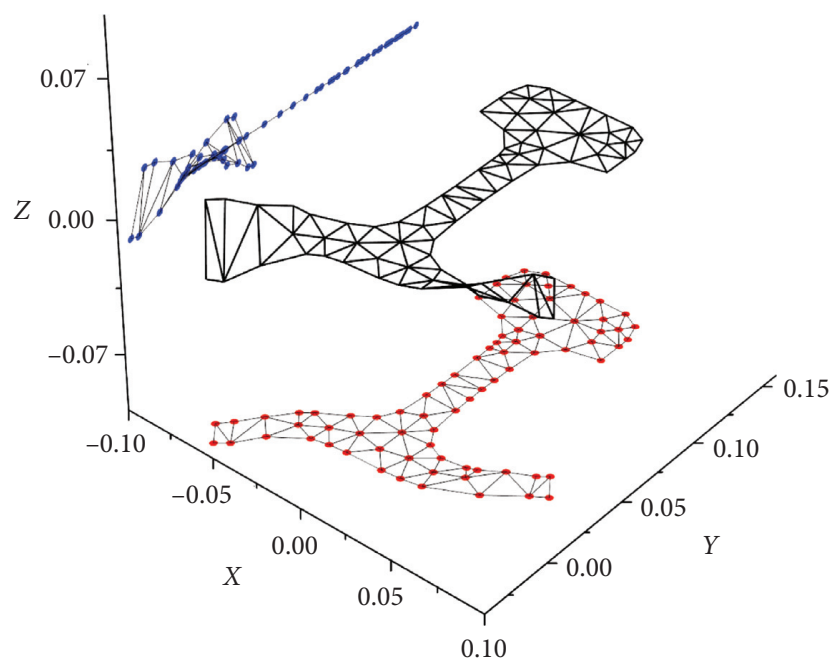

(a)

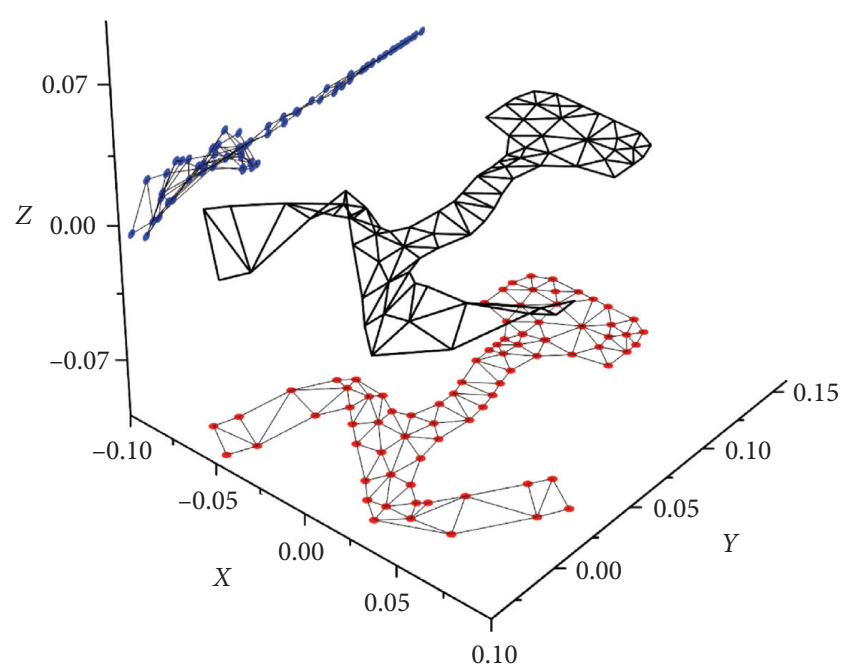

(b)

Figure 4: The wave structures with the center frequency $90 \mathrm{kHz}$. (a) Mode $v_{1}$ with $c_{\mathrm{p}}=2534 \mathrm{~m} / \mathrm{s}$ and $c_{\mathrm{g}}=3340 \mathrm{~m} / \mathrm{s}$. (b) Mode $v_{2}$ with $2950 \mathrm{~m} / \mathrm{s}$ $c_{\mathrm{g}}=3190 \mathrm{~m} / \mathrm{s}$.

The wave structures of Mode $V_{1}$ and Mode $V_{2}$ with the center frequency $90 \mathrm{kHz}$ are demonstrated in Figure 4. It demonstrates the distribution characteristics of the displacement in the $X Y$ and $Y Z$ planes. The vertical displacement of each position in the cross section can be got in the $X Y$ plane. The region at the rail base with large vertical displacement is suitable for placing guided wave transducers. The longitudinal displacement can be obtained in the $Y Z$ planes. The longitudinal displacement can reflect the longitudinal propagation ability of guided waves at various positions of the rail. The larger the longitudinal displacement, the stronger the propagation ability of guided waves. And this also denotes that the vibration attenuation is exceedingly slow. Figure 4(a) demonstrates that the node elements at the bottom edge ( $R 1$ in Figure 1) possesses large vertical displacement and longitudinal displacement. Figure 4(b) demonstrates that the obvious vertical and longitudinal displacements of the node elements occur at the center of the junction of the base edge and the flange ( $R 2$ in Figure 1). These results mean that it is indispensable to select the guided wave mode according to the detection position. The region at the bottom edge can be monitored by Mode $V_{1}$, while the junction of the base edge and the flange can be detected by Mode $V_{2}$.

3.2. Analysis of Propagation Progress of Vertical Mode at the Rail Base. The transient dynamic analysis model was established in ABAQUS, and the geometrical parameters and material parameters of the rail were the same as those in the SAFE method. The hexahedral elements were selected with the maximum mesh size of $0.002 \mathrm{~m}$, as demonstrated in Figure 5. The size of $0.002 \mathrm{~m}$ was smaller than the one-tenth of the wavelength to be studied.

The center of the flange at the rail base was selected as the excitation area. According to Figure 3, group velocity and phase velocity of guided wave in vertical mode tend to be stable when the excitation frequency is greater than $80 \mathrm{kHz}$.

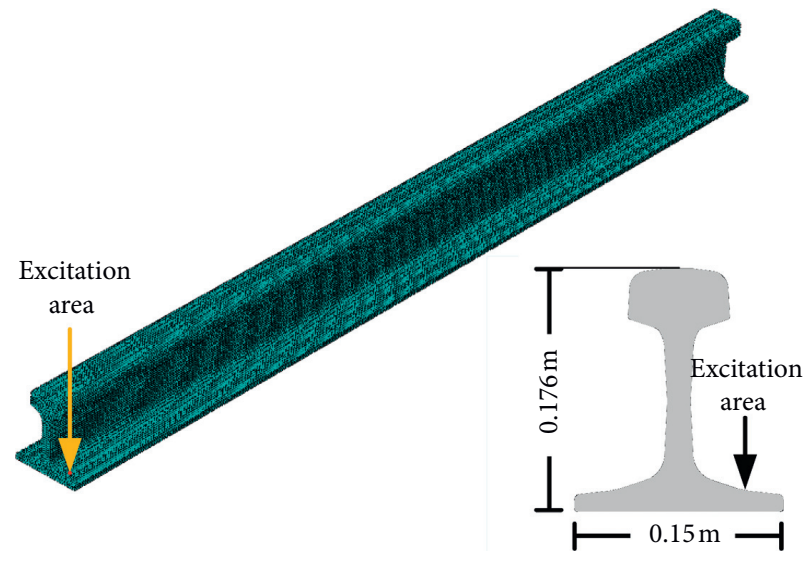

FIgURE 5: Finite element model of CHN60 rail.

Hence, the 5-period cosine rising signal with a central frequency of $90 \mathrm{kHz}$ was selected as the excitation signal of Mode $V_{1}$ with the sampling interval $\left(\Delta t=10^{-7} \cdot \mathrm{s}\right)$. Their time-history curve and spectrum are demonstrated in Figure 6.

Figure 7 demonstrates the propagation process of guide wave which was generated by vertical excitation at rail base. At $0.2 \mathrm{~ms}$, the wave packets contain multiple modes. The target mode is not separated. At $0.6 \mathrm{~ms}$, mode separation occurs. The energy of the wave packet with the faster speed concentrates at the edge of the rail base, demonstrated as region $A$. And the energy of the wave packet with the slower speed is distributed on the cross section of the whole rail base.

The wave structures at $A$ and $B$ are demonstrated in Figures $8(\mathrm{~b})$ and $8(\mathrm{~d})$, respectively. Because the excitation was loaded at the one side of the rail base, there are obvious vertical displacements at the same side in Figures 8(b) and $8(\mathrm{~d})$. The wave structures of Mode $V_{1}$ and Mode $V_{2}$ are demonstrated in Figures 8(a) and 8(c), respectively. The deformations at the same sides in Figures 8(a) and 8(b) are 


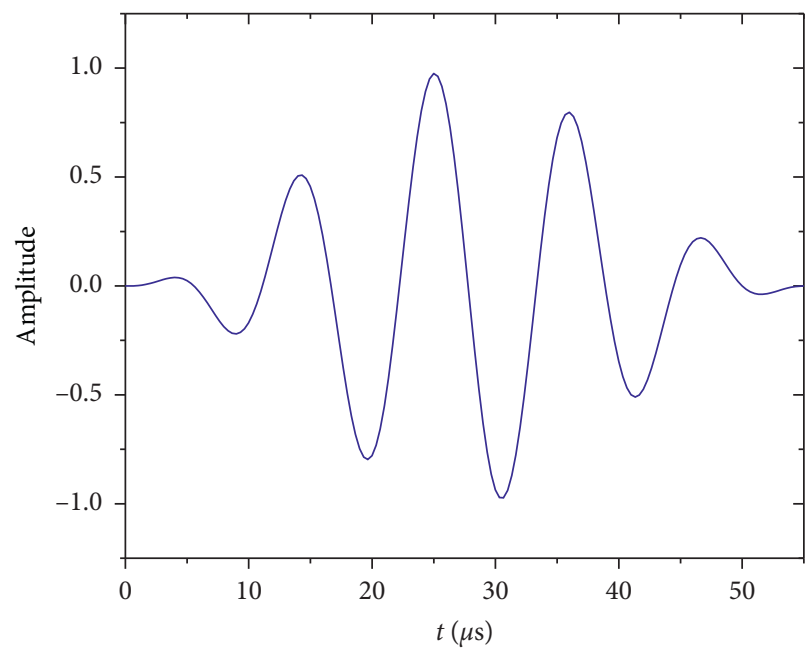

(a)

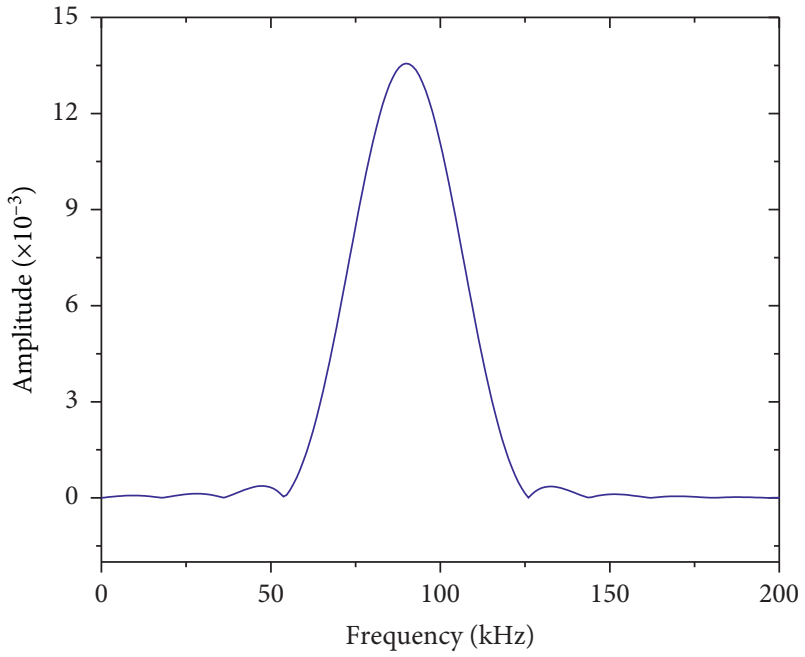

(b)

FIgure 6: The excitation signal with a central frequency of $90 \mathrm{kHz}$. (a) The time-history curve. (b) The spectrum.

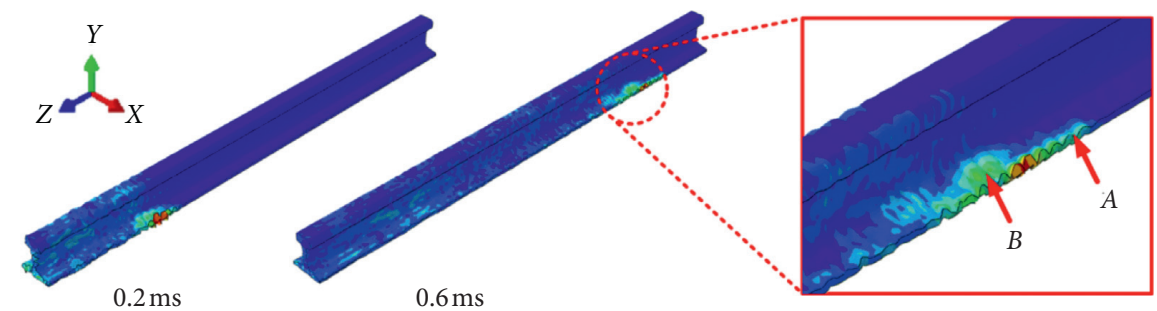

Figure 7: The propagation characteristics of guided wave by vertical excitation at rail base.

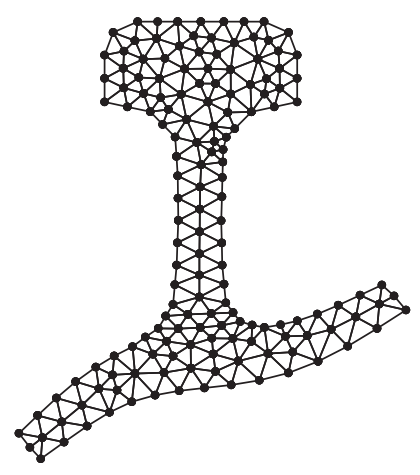

(a)

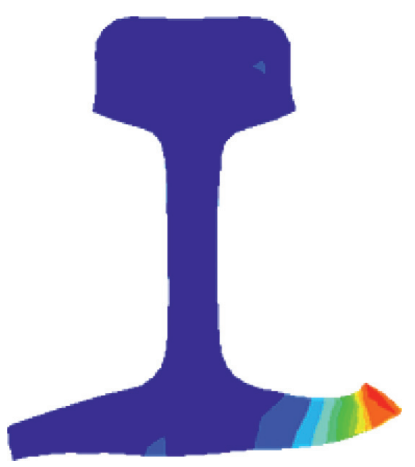

(b)

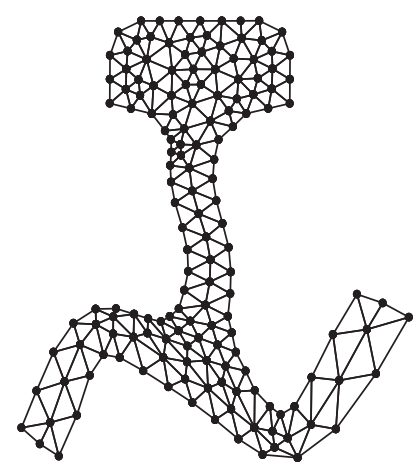

(c)

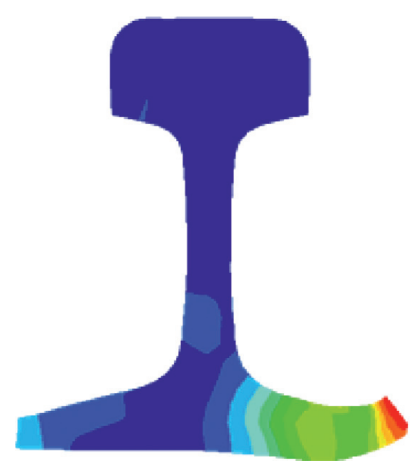

(d)

Figure 8: Deformation comparison at the rail base from SAFE and FEM. (a) Mode $V_{1}$. (b) The cross section at $A$. (c) Mode $V_{2}$. (d) The cross section at $B$.

extremely similar, when the other sides are different because of no excitation. The deformation characteristics of the cross section at point $B$ are consistent with Mode $V_{2}$.

In Figure 4, the group velocity of Mode $V_{1}$ is about $3340 \mathrm{~m} / \mathrm{s}$ when the frequency is $90 \mathrm{kHz}$, and that of Mode $V_{2}$ is about $3190 \mathrm{~m} / \mathrm{s}$. The transducers were deployed at the $R 1$ and the $R 2$ (as demonstrated in Figure 1) in the same cross section, respectively. The receiving points were $1.5 \mathrm{~m}$ away from the excitation point. The vertical displacement curves at the receiving points are demonstrated in Figure 9. Their envelopes can be obtained by the Herbert transform. There are two maximums in Figure 9(a). It means that the wave packet contains no less than two modes. The group velocity of the faster is $3340 \mathrm{~m} / \mathrm{s}$, equal to the one of Mode $V_{1}$. The group velocity of the slower is $3177 \mathrm{~m} / \mathrm{s}$, which is equal to the one of Mode $V_{2}$. Figure 9(b) demonstrates there is one 


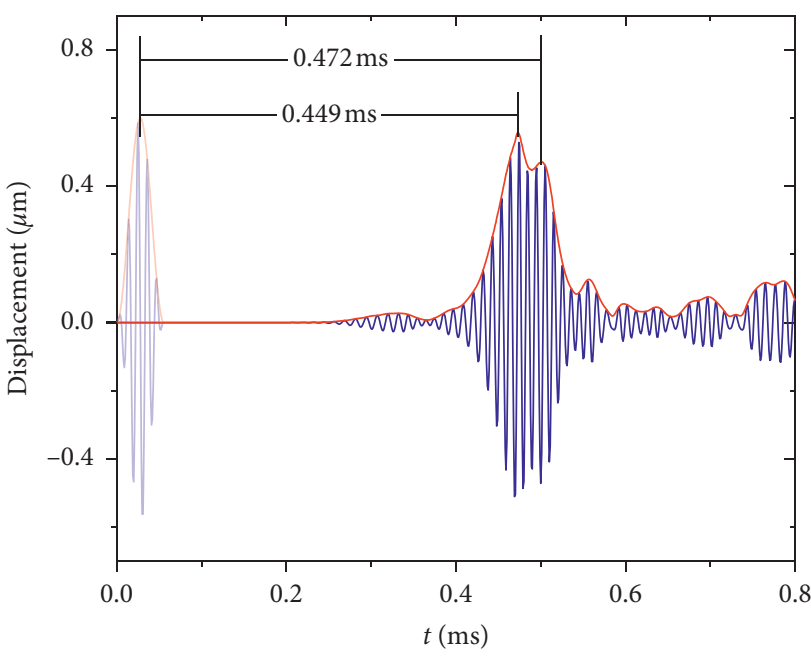

(a)

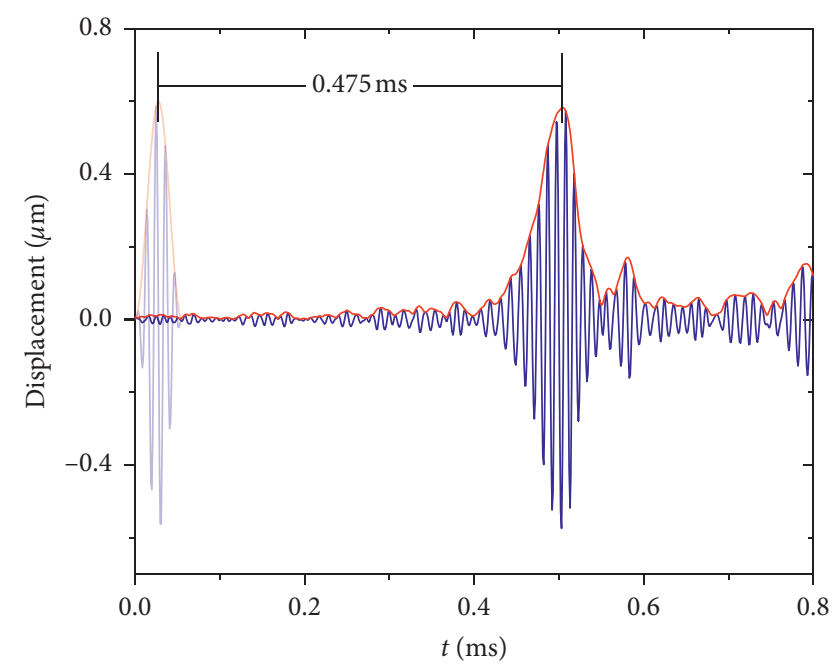

(b)

Figure 9: The vertical displacements at (a) $R 1$ and (b) $R 2$ as demonstrated in Figure 1.

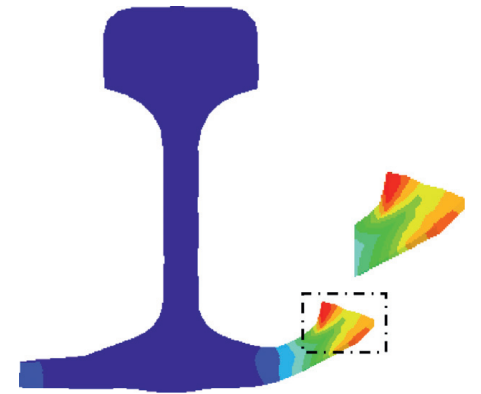

(a)

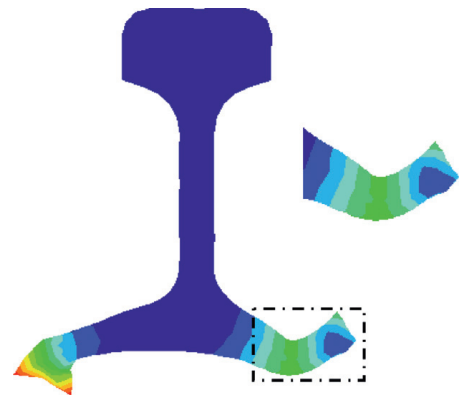

(d)

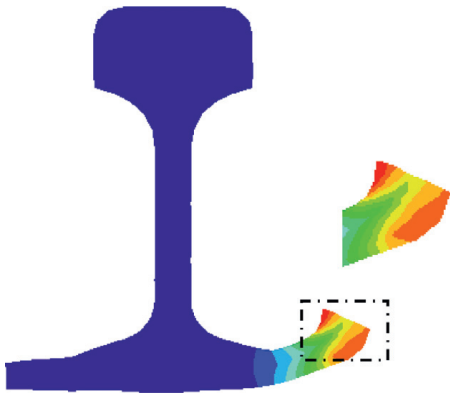

(b)

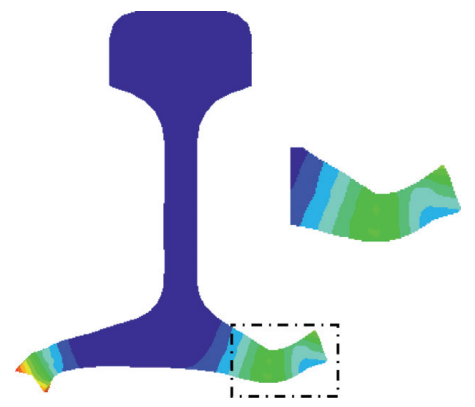

(e)

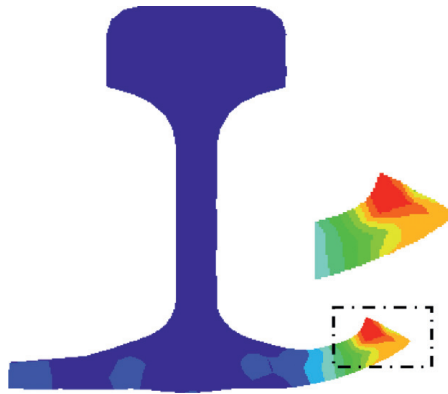

(c)

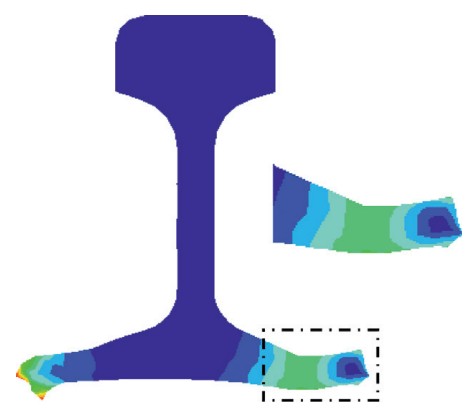

(f)

Figure 10: Deformation comparison at the rail base. (a-c) Mode $V_{1}$ at $3 \mathrm{~m}, 5 \mathrm{~m}$, and $7 \mathrm{~m}$, respectively. (d-f) Mode $V_{2}$ at $3 \mathrm{~m}, 5 \mathrm{~m}$, and $7 \mathrm{~m}$, respectively.

maximum. Its group velocity is about $3158 \mathrm{~m} / \mathrm{s}$. This mode can be considered as Mode $V_{2}$. These results are consistent with the aforementioned analysis. Modes $V_{1}$ and $V_{2}$ can be received at $R 1$ while only Mode $V_{2}$ can be received at $R 2$.

3.3. Analysis of Wave Attenuation. Take two mode waves at $3 \mathrm{~m}, 5 \mathrm{~m}$, and $7 \mathrm{~m}$ from the excitation position as an example to study the mode attenuation, as demonstrated in Figure 10. Because their velocities are constant, the arrival time of the same wave can be got precisely. Following the proposed algorithm in Section 2.2, the identifications of Modes $V_{1}$ and $V_{2}$ are as follows:

$$
\begin{aligned}
& \mathrm{ID}_{V 1,3 \mathrm{~m}}=001 \mathrm{~F} 801 \mathrm{~F} 80 \mathrm{FC} 007 \mathrm{E} 003 \mathrm{~F} \\
& \mathrm{ID}_{V 1,5 \mathrm{~m}}=00078007803 \mathrm{C} 003 \mathrm{E} 000 \mathrm{~F} \\
& \mathrm{ID}_{V 1,7 \mathrm{~m}}=00038003803 \mathrm{C} 001 \mathrm{E} 0007 \\
& \mathrm{ID}_{V 2,3 \mathrm{~m}}=\mathrm{F} 007 \mathrm{~F} 007 \mathrm{E} 03 \mathrm{~F} 001 \mathrm{FC} 00 \mathrm{~F} \\
& \mathrm{ID}_{V 2,5 \mathrm{~m}}=\mathrm{FC} 0 \mathrm{FF} 807 \mathrm{~F} 03 \mathrm{FC} 01 \mathrm{FE} 01 \mathrm{~F}
\end{aligned}
$$


$\mathrm{ID}_{V 2,7 \mathrm{~m}}=\mathrm{FE} 0 \mathrm{FFC} 0 \mathrm{FF} 87 \mathrm{FE} 03 \mathrm{FF} 01 \mathrm{~F}$

The hamming distances are as follows:

$$
\begin{aligned}
& D\left(\operatorname{ID}_{V 1,3 \mathrm{~m}}, \mathrm{ID}_{V 1,5 \mathrm{~m}}\right)=7 \\
& D\left(\mathrm{ID}_{V 1,5 \mathrm{~m}}, \mathrm{ID}_{V 1,7 \mathrm{~m}}\right)=10 \\
& D\left(\mathrm{ID}_{V 2,3 \mathrm{~m}}, \mathrm{ID}_{V 2,5 \mathrm{~m}}\right)=7 \\
& D\left(\mathrm{ID}_{V 2,5 \mathrm{~m}}, \mathrm{ID}_{V 2,7 \mathrm{~m}}\right)=13
\end{aligned}
$$

The hamming distances of the two mode waves between $3 \mathrm{~m}$ and $5 \mathrm{~m}$ from the excitation position are equal to 7 . This means that both waves have the same attenuation. The hamming distance of Mode $V_{2}$ between $5 \mathrm{~m}$ and $7 \mathrm{~m}$ is equal to 13 and the largest. This means that Mode $V_{2}$ decays slightly faster than Mode $V_{1}$ at this range. The hamming distances show that both mode waves can be detected within $5 \mathrm{~m}$ from the excitation position.

In practical application, the receiving point should be at a range from the excitation point. If they are too close, the modes may not be completely separated. The wave packet may contain different modes of guided waves. If they are too far, the energy may decay too much. And the mode cannot be detected. Besides, when the energy of the target mode is much higher than that of other modes, the received guide wave can be approximately considered as a single mode guide wave. It can be identified by analyzing the wave structure of the target mode, the excitation, and the receiving position reasonably.

\section{Experiment Research}

In the experiments, the PZT was used to motivate and receive ultrasonic guided wave. And firstly, signal generator RIGOL DG1022U generated waveform signals. Then, the signals were amplified by the ATA-2042 power amplifier. Finally, the amplified signals drove the PZT to generate ultrasonic guided waves. In the receiving part, the PZT was used to convert the mechanical signal to the electrical signal, which was output to the PC end through the oscilloscope PICO 5444D for subsequent analysis. The synchronization function of the signal generator was used to get the excitation moments. Figure 11 demonstrates how the devices connect together.

4.1. Mode-Identified Experiment. As the aforementioned analysis, there are Mode $V_{1}$ and Mode $V_{2}$ at the rail base between 0 and $100 \mathrm{kHz}$. The difference of their group velocities is small when the frequency is near $90 \mathrm{kHz}$. In addition, the receiving signals can be contaminated by noise. Hence, it is arduous to accurately get the time corresponding to the peak value. This may result in a big deviation by detecting group velocity. Therefore, the modes were identified by measuring the phase velocity.

It is indispensable to obtain the information of the timespace dimension of the guided waves to measure the phase velocity. A single probe was used many times as the receiving probe. After each measurement, the receiving probe would be shifted along the wave propagation direction by a certain

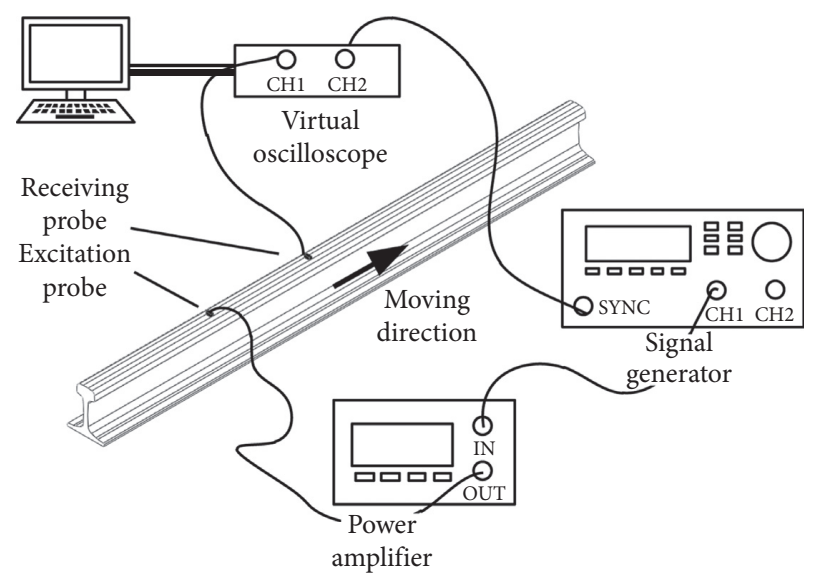

FIGURE 11: The device connection diagram for measuring the phase velocity.

distance. The wavenumber-frequency relationship was obtained by the 2D-FFT.

The excitation probe and the receiving probe were placed at the middle of the flange of the rail base, as demonstrated in Figure 12. The excitation signal was a sinusoidal wave of $90 \mathrm{kHz}$ with five consecutive cycles. The initial position of the receiving probe was $0.5 \mathrm{~m}$ away from the excitation probe. Then, the receiving probe was moved $0.005 \mathrm{~m}$ away from the last measurement after each measurement. In the experiment, the interval between the sampling points was $0.005 \mathrm{~m}$, and the corresponding spatial sampling rate was $200 \mathrm{Sa} / \mathrm{m}$. The sampling interval was $2 \times 10^{-7} \mathrm{~s}$, and the corresponding sampling rate was $5 \mathrm{MSa} / \mathrm{s}$. A total of 20 sets of data were measured.

Figure 13 demonstrates the history waveforms at the three measurement points, which were $0.51 \mathrm{~m}, 0.57 \mathrm{~m}$, and $0.63 \mathrm{~m}$ away from the excitation point. It is easy to find a clear wave packet. Figure 14 demonstrates the wavenumber-frequency contour obtained by 2D-FFT. It consists of wavenumber, frequency, and amplitude. The shadow in Figure 14 represents the wavenumber-frequency relationship of the receiving mode at the rail base. It can be seen that the experimental results are consistent with the dispersion relationship of Mode $V_{2}$. Mode $V_{2}$ is the dominant mode among the vertical modes at the center of the flange at the rail base. Its energy is concentrated at the rail base. And its longitudinal displacement and vertical displacement are also large. These reveal that the propagation characteristics of Mode $V_{2}$ are suitable for the defect detection at the rail base.

4.2. Defect Detection Experiment at the Rail Base. A crack was made at the rail base, with the transverse depth of about $0.023 \mathrm{~m}$, the vertical depth of about $0.01 \mathrm{~m}$, and the longitudinal width of about $0.002 \mathrm{~m}$. The damage area was about $15 \%$ of the cross section area, as demonstrated in Figure 15. The excitation signal was a 5-period cosine rising signal, with the central frequency of $90 \mathrm{kHz}$. The excitation probe and receiving probe were arranged symmetrically on both sides of the damage at the center of the rail base and the flange. 


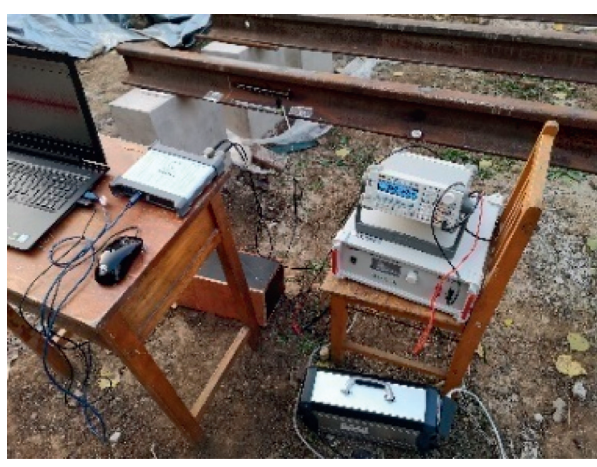

(a)

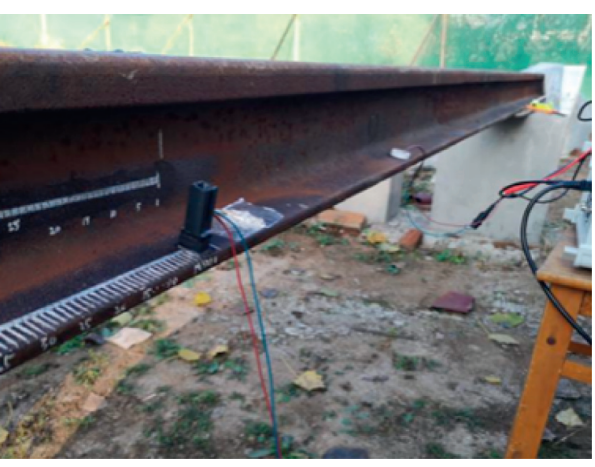

(b)

FIgURE 12: The experiment field for measuring the phase velocity.

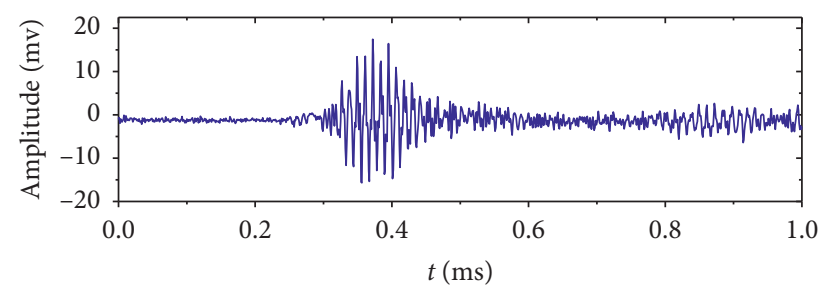

(a)

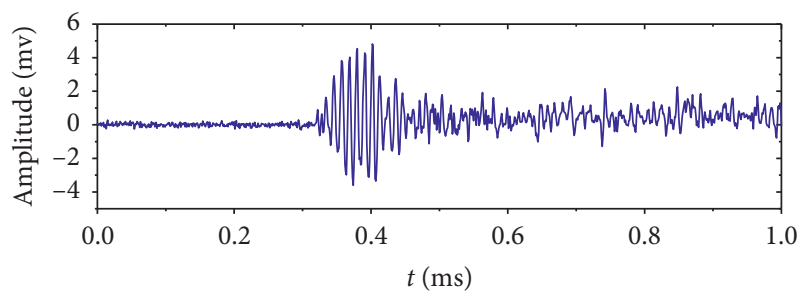

(b)

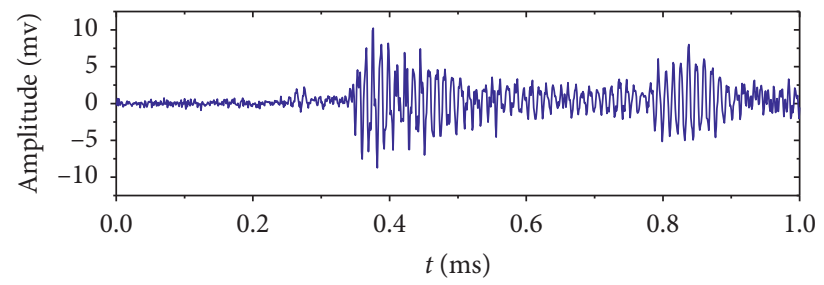

(c)

Figure 13: The history waveforms at the (a) $0.51 \mathrm{~m}$, (b) $0.57 \mathrm{~m}$, and (c) $0.63 \mathrm{~m}$ away from the excitation point.

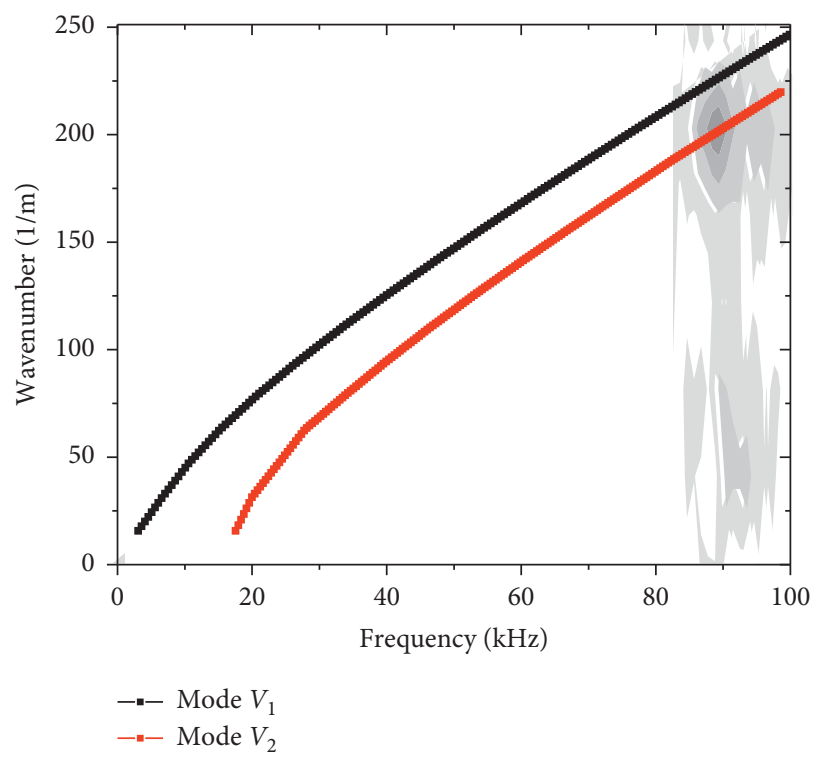

FIGURE 14: The wavenumber-frequency relationship by the 2D-FFT. 


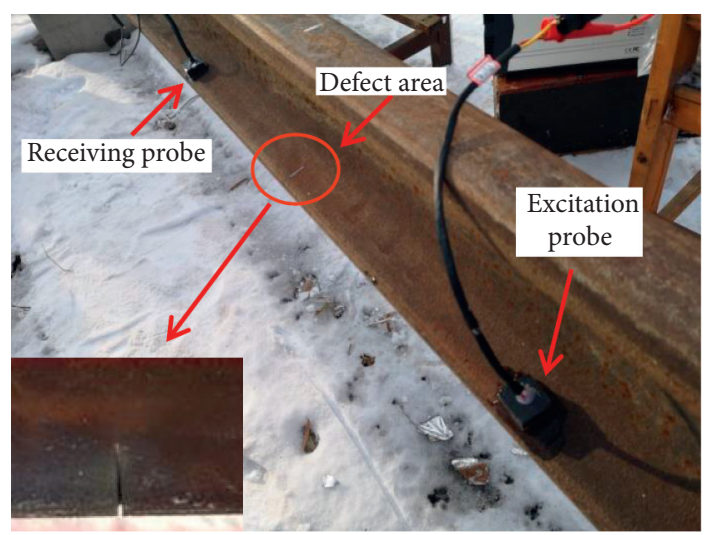

Figure 15: The defect detection experiment at the rail base.

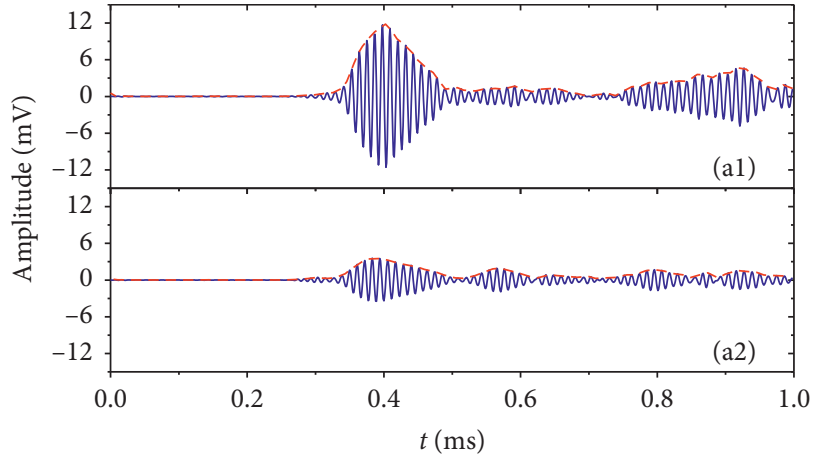

(a)

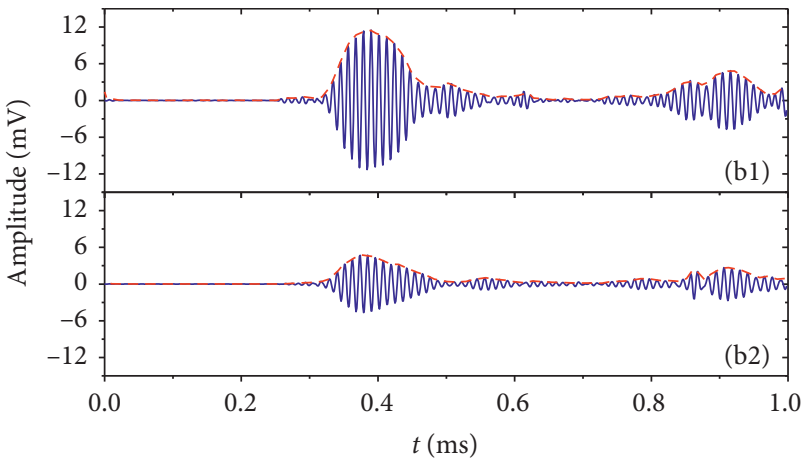

(b)

FIGURE 16: The receiving signal with (a1) no defect, (a2) transverse defect in the first group, (b1) no defect, and (b2) transverse defect in the second group.

According to the aforementioned analysis, Mode $V_{2}$ is the dominant mode excited vertically at this position. Two groups of experiments were designed for comparison. In the first one, the excitation probe and the receiving probe were arranged on both sides of the damage; they were $0.3 \mathrm{~m}$ away from the damage. In the second one, the excitation probe and the receiving probe were arranged on both sides of the damage, and they were $0.6 \mathrm{~m}$ away from the damage. Moreover, each group was set up with a nondamage control group for comparison.

Figure 16 demonstrates the receiving signal. The peak value in Figure 16 (a1) is $11.9 \mathrm{mV}$. The peak value in Figure 16 (b1) is $11.6 \mathrm{mV}$. There is no obvious difference. It can be seen that the energy of Mode $V_{2}$ does not decrease significantly from $0.3 \mathrm{~m}$ to $0.6 \mathrm{~m}$. The peak value in Figure 16 (a2) is $3.6 \mathrm{mV}$. This means that the defect makes the energy reduce by $69.7 \%$ in the first group. The peak value in Figure 16 (b2) is $4.8 \mathrm{mV}$. The defect makes the energy decrease by $58.6 \%$ in the second group. In both groups, the amplitudes of Mode $V_{2}$ significantly decrease under damage conditions, indicating the feasibility of using Mode $V_{2}$ to detect transverse defects at the rail base.

\section{Conclusion}

Ultrasonic guided wave can travel a long distance, and it has attracted growing attention in the nondestructive testing of rails. This paper studies the propagation of the guided waves in the vertical direction at the rail base. The SAFE method is used to calculate the dispersion and wave structure characteristics of the guided wave. It demonstrates that Mode $V_{1}$ and Mode $V_{2}$ with the center frequency of $90 \mathrm{kHz}$ are suitable as the selected modes, and their energy concentrates at the rail base. Besides, the propagation of the guide waves excited vertically at the rail base is studied by FEM. The results demonstrate that Mode $V_{1}$ propagates only at the base edge, while Mode $V_{2}$ can propagate at the base flange. A wave attenuation algorithm based on mean is proposed and can quantify the attenuation of both waves. Both waves have weak attenuations and can be detected within $5 \mathrm{~m}$ from the excitation position.

Experiments are conducted on the rail experiment platform. And guided wave modes are identified by detecting phase velocity in order to verify the simulation results. The results demonstrate that the dominant mode of 
guided wave excited at the center of the rail base and the flange is Mode $V_{2}$, consistent with the simulation results. The defect at the rail base can be detected by the change of the amplitudes of Mode $V_{2}$. Mode $V_{2}$ can be selected as the suitable mode for the nondestructive detection of rail base.

\section{Data Availability}

The data used to support the findings of this study are included within the article.

\section{Conflicts of Interest}

The authors declare that they have no conflicts of interest regarding the publication of this paper.

\section{Acknowledgments}

The authors gratefully acknowledge the support from the National Natural Science Foundation of China (NSFC) (Grant nos. 51705340 and 51805337) and the Natural Science Foundation of Liaoning Province (Grant nos. 20170540745 and 20180551124).

\section{References}

[1] M. Bogdevicius, R. Zygiene, G. Bureika, and S. Dailydka, “An analytical mathematical method for calculation of the dynamic wheel-rail impact force caused by wheel flat," Vehicle System Dynamics, vol. 54, no. 5, pp. 689-705, 2016.

[2] H.-B. Yun, K. C. Lee, Y. J. Park, and D.-K. Jung, "Rail neutral temperature monitoring using non-contact photoluminescence Piezospectroscopy: a field study at high-speed rail track," Construction and Building Materials, vol. 204, pp. 357-370, 2019.

[3] Q. Feng, W. Wei, X. Sheng, Q. Liu, and X. Lei, "Vertical vibration analysis of temperature-stressed CWR using wave number finite element method," International Journal of Rail Transportation, vol. 6, no. 2, pp. 131-144, 2018.

[4] G.-D. Yue, Z. Xu, L. Wang, C. Liu, and T. Ren, "WSN-based vibration characteristic research for various railway track structures for pattern classification," International Journal of Pattern Recognition and Artificial Intelligence, vol. 30, no. 10, Article ID 1650020, 2016.

[5] G. Liu, J. Philtron, Y. Zhu, J. L. Rose, and M. Han, "Detection of fundamental shear horizontal guided waves using a surfacebonded chirped-fiber-Bragg-grating Fabry-Perot interferometer," Journal of Lightwave Technology, vol. 36, no. 11, pp. 2286-2294, 2018.

[6] J. F. Doyle, "Determining the size and location of transverse cracks in beams," Experimental Mechanics, vol. 35, no. 3, pp. 272-280, 1995.

[7] A. Apostoloudia, E. Douka, L. J. Hadjileontiadis, I. T. Rekanos, and A. Trochidis, "Time-frequency analysis of transient dispersive waves: a comparative study," Applied Acoustics, vol. 68, no. 3, pp. 296-309, 2007.

[8] P. W. Loveday and C. S. Long, "Long range guided wave defect monitoring in rail track," in Proceedings of the AIP Conference. American Institute of Physics, pp. 179-185, Surakarta, Indonesia, 2014.

[9] C. S. Long and P. W. Loveday, "Prediction of guided wave scattering by defects in rails using numerical modelling," in
Proceedings of the AIP Conference. American Institute of Physics, pp. 240-247, Surakarta, Indonesia, 2014.

[10] M. I. Abdelrahman and B. Gralak, "Modal analysis of wave propagation in dispersive media," Physical Review A, vol. 97, no. $1,2017$.

[11] S. Sengupta, D. R. Jackson, and S. A. Long, "Modal analysis and propagation characteristics of leaky waves on a 2-D periodic leaky-wave antenna," IEEE Transactions on Microwave Theory and Techniques, vol. 66, no. 3, pp. 1-11, 2018.

[12] G. Capuano, M. Ruzzene, and J. J. Rimoli, "Modal-based finite elements for efficient wave propagation analysis," Finite Elements in Analysis and Design, vol. 145, pp. 10-19, 2018.

[13] Z. Su, L. Ye, and Y. Lu, "Guided Lamb waves for identification of damage in composite structures: a review," Journal of Sound \& Vibration, vol. 295, no. 3-5, pp. 753-780, 2006.

[14] M. Mitra and S. Gopalakrishnan, "Guided wave based structural health monitoring: a review," Smart Materials and Structures, vol. 25, no. 5, Article ID 053001, 2016.

[15] A. B. Noel, A. Abdaoui, T. Elfouly et al., "Structural health monitoring using wireless sensor networks: a comprehensive survey," IEEE Communications Surveys \& Tutorials, vol. 19, no. 3, pp. 1403-1423, 2017.

[16] G. Giunta, Y. Koutsawa, S. Belouettar, and A. Calvi, “A dynamic analysis of three-dimensional functionally graded beams by hierarchical models," Smart Structures and Systems, vol. 13, no. 4, pp. 637-657, 2014.

[17] D. A. Ramatlo, D. N. Wilke, and P. W. Loveday, "Development of an optimal piezoelectric transducer to excite guided waves in a rail web," NDT \& E International, vol. 95, pp. 72-81, 2018.

[18] L. Gavrić, "Computation of propagative waves in free rail using a finite element technique," Journal of Sound and Vibration, vol. 185, no. 3, pp. 531-543, 1995. 